\title{
Study of Methylene Blue Degradation by Gold Nanoparticles Synthesized within Natural Zeolites
}

\author{
Ericka Rodríguez León, ${ }^{1}$ Eduardo Larios Rodríguez, ${ }^{2}$ César Rodríguez Beas, ${ }^{1}$ \\ Germán Plascencia-Villa, ${ }^{3}$ and Ramón Alfonso Iñiguez Palomares ${ }^{1}$ \\ ${ }^{1}$ Departamento de Física, Universidad de Sonora, Rosales y Transversal, 83000 Hermosillo, SON, Mexico \\ ${ }^{2}$ Departamento de Ingeniería Química y Metalurgia, Universidad de Sonora, Rosales y Transversal, 83000 Hermosillo, SON, Mexico \\ ${ }^{3}$ Department of Physics and Astronomy, The University of Texas at San Antonio, One UTSA Circle, San Antonio, TX 78249, USA
}

Correspondence should be addressed to Ramón Alfonso Iñiguez Palomares; ramonalfonso.iniguez@gmail.com

Received 28 March 2016; Accepted 28 April 2016

Academic Editor: Xuping Sun

Copyright (C) 2016 Ericka Rodríguez León et al. This is an open access article distributed under the Creative Commons Attribution License, which permits unrestricted use, distribution, and reproduction in any medium, provided the original work is properly cited.

\begin{abstract}
We carried out the in situ synthesis of gold nanoparticles inside a natural clinoptilolite-type zeolite matrix, using ascorbic acid as reducing agent. The microstructure of both zeolite and zeolite-gold nanocomposite was characterized by X-ray diffraction (XRD), Scanning Electron Microscopy (SEM), Scanning Transmission Electron Microscopy (STEM), and Energy-Dispersive X-ray Spectroscopy (EDS) techniques. Size distribution as assessed by STEM indicated that $60 \%$ of gold nanoparticles measured less than $2.5 \mathrm{~nm}$. Determination of the surface area by the BET method revealed a specific value of $27.35 \mathrm{~m}^{2} / \mathrm{g}$. The catalytic activity of zeolitegold regarding methylene blue degradation under different light-exposing conditions was evaluated by UV-Vis spectroscopy. The results indicated that $50 \%$ degradation was achieved in only $11 \mathrm{~min}$ in presence of sunlight. This reaction was faster in comparison with those obtained using a white LED light. A notable aspect of this study is that catalysis was carried out without the addition of any strong reducing agents, such as sodium borohydride $\left(\mathrm{NaBH}_{4}\right)$.
\end{abstract}

\section{Introduction}

Currently, the serious air, ground, and water pollution problems pose enormous challenges in the area of catalysis [1]. In this regard, the catalytic processes occurring naturally in our environment should serve as an example for designing and developing new catalysts. The development of these new catalysts pursues to improve their activity and selectivity while decreasing by-product generation, as well as that of other pollutants. Catalysis classification is based on the interaction between the phases comprising the system. Heterogeneous catalysis takes place when a solid phase (macroscopic catalyst) interacts with a liquid phase system. Regarding homogenous catalysis, the interaction occurs between two liquid phases (at the macroscopic level). Currently, the most used catalysts in industry are those of the heterogeneous type [2]; they stand out for several reasons such as selectivity, their long lifespan, and their ability to be reused. Because of these reasons, the most suitable supporting materials for heterogeneous catalyst manufacture are those possessing high porosity and consequently a high surface area.

Catalytically active material is laid on surfaces such as natural and synthesized aluminosilicate, metal oxides, and activated carbon. All of these are commonly used for this purpose $[3,4]$. These materials function as support for nanocatalysts and exert a heterogeneous catalytic effect, thereby creating a synergy by inducing an interaction [5] between the components (zeolites and nanoparticles, in this case). This renders the catalytic processes even more efficient. In addition, nanomaterial confinement produces different effects on the catalytic response displayed by the materials laid on the supporting surface [6-8].

On the other hand, several industries such as those processing textiles, paper, and leather use dyes in order to add color to their respective products. They also consume 
considerable amounts of water and consequently they induce massive pollution [9]. Particularly, the cationic compound methylene blue (MB) is the most used dye in order to process different types of textiles such silk, cotton, and wool [1012]. This dye represents a health hazard for both humans and animals. Therefore, it must be removed from the water that has been already polluted by industrial activity $[13,14]$. Among the alternatives for dye-polluted water remediation, adsorption methods have additional advantages regarding other processes as their cost is low, they are easy to operate, and they do not release toxic substances into the process [15].

In this work, a natural zeolite (clinoptilolite type) was used as supporting material [16-18]. It has a complex molecular formula: $(\mathrm{Na}, \mathrm{K}, \mathrm{Ca}) 2-3 \mathrm{Al}_{3}(\mathrm{Al}, \mathrm{Si}) 2 \mathrm{Sil}_{3} \mathrm{O}_{36} \cdot 12 \mathrm{H}_{2} \mathrm{O}$, and it possesses a nanopore array and a high thermal stability, being thus ideal for this purpose. Zeolites are materials possessing pores ranging between 0.5 and $2 \mathrm{~nm}$ [19]. Nanometric-sized metallic gold was used as active material whereas ascorbic acid was the reducing agent in order to trigger gold ( $\mathrm{Au}$ ) nanoparticles synthesis [20-22].

A study on the catalytic effect of the obtained zeolite$\mathrm{Au}$ nanocomposite (ncomp zeo-Au) was carried out using MB photodegradation as experimental model [15, 23, 24]. Since illumination plays a major role in catalytic processes [25-27], the experiments were conducted using two different light sources: (a) a LED lamp as source of white light and (b) sunlight. In order to ascertain the contribution of the zeolite matrix devoid of gold nanoparticles on MB catalytic degradation, two experiments using natural zeolite (clinoptilolite) were carried out under the same illumination conditions as described above. Moreover, several authors use the reducing agent $\mathrm{NaBH}_{4}$ as complement in order to accelerate the catalytic process by directly adding it into the solution to be bleached [28, 29]. However, the use of this compound increases the cost of the catalytic process and it contributes to additional water pollution. Considering all of the above, in this paper we show that gold nanomaterials synthesized within the zeolite matrix have an efficient catalytic activity on $\mathrm{MB}$ photodegradation, without needing the addition of yet another compound in order to accelerate catalysis.

\section{Materials and Methods}

2.1. Materials. All reagents were of $\mathrm{AR}$ grade. $\mathrm{HAuCl}_{4}$ and ascorbic acid were purchased from Sigma Aldrich (99\% pure). 18.2 M $\Omega$ Milli-Q water was used throughout the experiment. Natural zeolite was a gift from the San Francisco (SF) mine in San Luis Potosi (Mexico).

2.2. Catalyst Preparation. Deposition was the chosen method to prepare the catalyst. In this procedure, the metal salt (aqueous $\mathrm{HAuCl}_{4}$ ) was incorporated by adsorption into the supporting material suspension and this occurred because of the interaction between the ions from the metallic compound and the active sites on the supporting material. Solvent excess may be removed by filtration or decantation. The interaction between the active component and the carrier is due to ion exchange between the supporting material

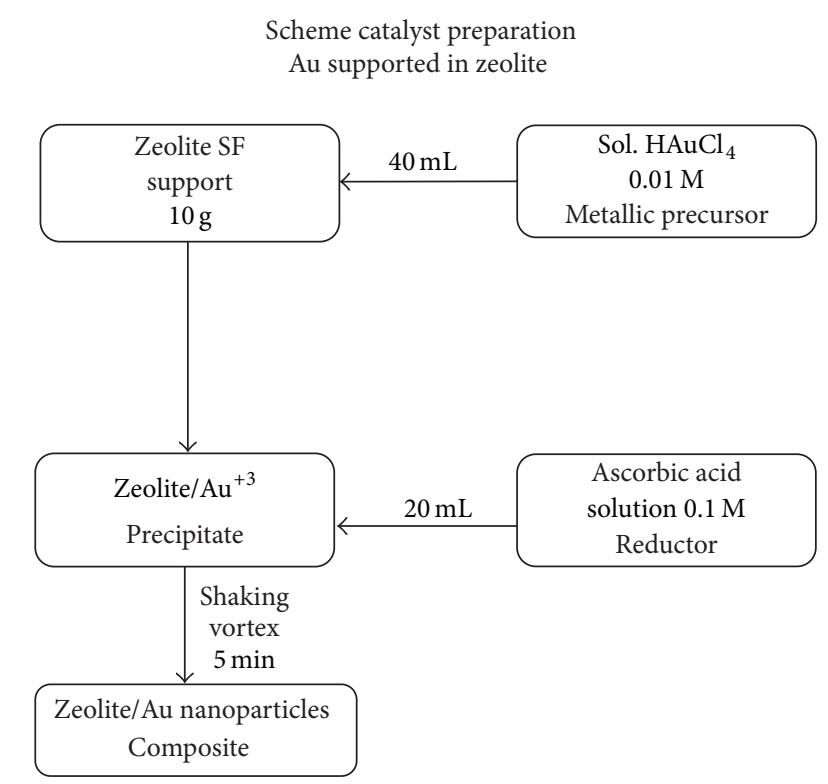

FIGURE 1: Scheme of the deposition method for catalyst preparation: Au nanoparticles supported in zeolites.

and the cationic species $\left(\mathrm{Au}^{3+}\right)$ in the solution. This catalyst preparation method has the advantage of producing low catalyst charges and also, through the interaction produced by adsorption, it stabilizes the metallic component preventing further catalyst synthesis. Thus, small catalyst particles appropriately dispersed on the surface of the supporting material were obtained. All of this renders this method suitable to perform the deposition of noble metals. The elevated cost of the catalyst is concomitantly reduced as small percentages are used while achieving a considerable dispersion on the supporting material's surface. Figure 1 shows the synthesis scheme.

Ten grams of zeolite, previously ground in an agate mortar, was placed in a $100 \mathrm{~mL}$ glass flask. Initially, a $40 \mathrm{~mL}$ volume of the metallic precursor $\left(0.01 \mathrm{M} \mathrm{HAuCl}_{4}\right)$ was added and the mixture was homogenized by vortexing for $5 \mathrm{~min}$ at $2400 \mathrm{rpm}$. The sample was stored for $1 \mathrm{~h}$ to allow the impregnation of the zeolite and the supernatant (still turbid) is removed after this period. Afterwards, $10 \mathrm{~mL}$ of reducing agent (ascorbic acid $0.1 \mathrm{M}$ ) was added in order to induce precipitation. The obtained dispersion was vortexed at $2400 \mathrm{rpm}$ for $5 \mathrm{~min}$ to ensure homogenization. The sample was covered with aluminum foil to avoid ascorbic acid photooxidation. The recovered solid was placed in a glass Petri dish and subsequently dried in an oven at $55^{\circ} \mathrm{C}$ for 24 hours. The material removed from the oven was crushed again in an agate mortar to perform the nanostructural study and to evaluate its catalytic activity. The net gold percentage in the zeo-Au composite was $0.7 \%$ (normalized weight).

\section{Results and Discussion}

3.1. X-Ray Diffraction. Both the ncomp zeo-Au system and the zeolite matrix were characterized by X-ray diffraction 


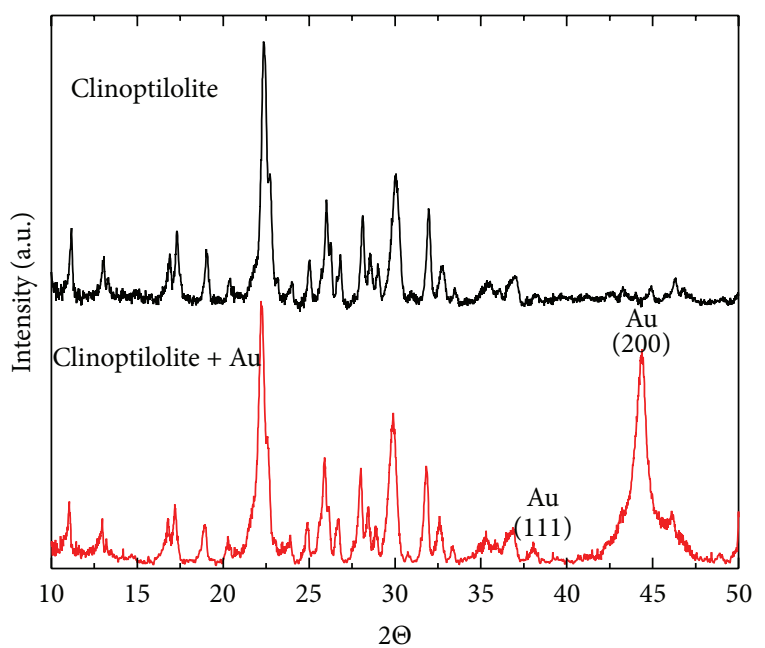

Figure 2: Comparison of X-ray diffraction patterns for natural zeolites and ncomp zeo-Au.

using PANalytical Empyrean Series 2 equipment possessing a Pixcel $^{3 \mathrm{D}}$ high-resolution detector. The experiments were run from 10 to 80 degrees with a $0.013^{\circ}$ step, a $60 \mathrm{kV}$ operating voltage, and $\mathrm{CuK} \alpha(\lambda=1.54060 \AA)$.

In Figure 2 the $\mathrm{X}$-ray diffraction patterns of pure zeolite and zeo-Au are observed. The software X-Pert High Score (PANalytical) was used for analysis and identification of the crystalline phases. The results indicate that the obtained diffractogram for the pure zeolite sample corresponds to the crystalline structure of clinoptilolite, having the molecular formula $\mathrm{H}_{42.64} \mathrm{Al}_{6} \mathrm{Ca}_{1.24} \mathrm{~K}_{1.76} \mathrm{Mg}_{0.2} \mathrm{Na}_{1.84} \mathrm{O}_{93.32} \mathrm{Si}_{30}$ and identified with the data card numer 98-010-0096 in the Inorganic Crystal Structure Database (ICSD). Besides clinoptilolite, in the diffractogram obtained for the zeo-Au sample, two peaks at $38.15^{\circ}$ and $44.37^{\circ}$ were identified corresponding to the (111) and (200) family of planes of gold fcc structure, respectively (ICSD 98-004-4362).

3.2. Scanning Electron Microscopy (SEM). SEM was performed using a Hitachi microscope (5500 model). Samples were prepared by dispersion in water using an ultrasonic bath. Subsequently, a drop of the dispersed solution was laid on a copper grid and stored at room temperature to allow drying. The obtained SEM micrographs (Figure 3) showed Au supported in zeolite. The crystal morphology of zeolite is observed as well as the presence of particles of approximately $5-7 \mathrm{~nm}$ size homogeneously distributed on the surface. This observation suggests that these quasisphericalshaped particles are smaller regarding zeolite crystals. This will be confirmed by further tests.

After performing an EDS analysis, Figure 4(a) shows that, besides zeolite components such as aluminum ( $\mathrm{Al})$, silicon $(\mathrm{Si})$, and oxygen $(\mathrm{O})$, the $\mathrm{Au}$ signal is also present. The $\mathrm{Cu}$ signal corresponds to the copper grid used to place the sample. Signals derive from most of the swept surface (Figure 4(b)), showing that gold is evenly distributed on most of the zeolite's surface as shown in the mapping image (Figure 4(c)).
3.3. Scanning Transmission Electron Microscopy (STEM). To perform the STEM analysis, a JEM-ARM200F (JEOL, USA) microscope equipped with aberration corrector CEOS Cs operated at $80 \mathrm{kV}$ was used. To conduct this study, samples of ncomp zeo-Au were prepared by sectioning using an ultramicrotome. The sample powder, previously ground in an agate mortar, was embedded in $1 \mathrm{~mL}$ of epoxy resin (Epon-LX112/DDSA/NADIC Methyl/DMP-30) polymerized for $24 \mathrm{~h}$ at $60^{\circ} \mathrm{C}$ and it was subsequently sectioned using an ultramicrotome (Leica Reichert Ultracut $\mathrm{S}$ ), with a Diatome Ultra $45^{\circ} \mathrm{knife}$. The obtained sections were $90 \mathrm{~nm}$ thick.

Figure 5 shows dark field $(\mathrm{a}-\mathrm{d})$ and bright field (eh) STEM micrographs. These show the presence of quasispherical nanoparticles between 1 and $5 \mathrm{~nm}$ of diameter. In this study, particles under $5 \mathrm{~nm}$ are observed. However, this observation was not possible through the SEM study as its resolution is low. The presence of gold nanoparticles on the zeolite sections indicates the presence of gold within its structure. A higher magnification of the ncomp zeo-Au sample is shown in Figure 6. In the latter, gold nanoparticles appear in the zeolite structure. Size distribution of gold nanoparticles indicates that $60 \%$ of them measure less than $2.5 \mathrm{~nm}$.

3.4. Specific Surface Area. Specific surface area by the Brunauer-Emmett-Telle (BET) method was determined with Gemini VII 2390 (Micrometrics). The nitrogen adsorptiondesorption isotherms were acquired at $-196^{\circ} \mathrm{C}$. The natural zeolite showed a surface area of $21.90 \mathrm{~m}^{2} / \mathrm{g}$, whereas the ncomp zeo-Au presented a specific value of $27.35 \mathrm{~m}^{2} / \mathrm{g}$. This increment in the specific surface area in comparison with control sample was attributed to the functionalization of zeolite with Au nanoparticles. It is important to notice that the zeolites were not treated with any harsh acids nor high temperatures that in cases may increase the surface area of materials.

3.5. Photocatalysis. Photocatalysis is generated from the interaction between light and semiconductive or conductive nanoparticles $[23,30]$. The wavelengths associated with the photocatalytic activity of semiconductor nanoparticles are mainly those in the near UV region (as the case of $\mathrm{TiO}_{2}$ ), whereas the visible region is associated with the optical absorption of metal nanoparticles (e.g., gold and silver) and thus with their photocatalytic activity. In this work the catalytic activity of natural zeolite and ncomp zeo-Au was evaluated using MB as experimental model. The study was performed using UV-Vis spectroscopy (Perkin-Elmer Lambda 45) in the range between $900 \mathrm{~nm}$ and $200 \mathrm{~nm}$ in order to monitor optical absorption changes of a $\mathrm{MB}$ aqueous solution $(0.33 \mathrm{mM})$ through time. The studies were conducted under two different light-exposure conditions: (a) using a LED white light lamp (high brightness) (2000 lumens and $25 \mathrm{~W}$ ) and (b) under the sunlight. To study the catalytic activity, the samples were ground in an agate mortar in order to obtain a fine powder such as talc and they were placed in small bags made out of filter papers $(1.5 \mathrm{~cm} \times 1.5 \mathrm{~cm})$, which were subsequently sealed. The bags containing $0.15 \mathrm{~g}$ of sample were introduced in transparent glass containers with 


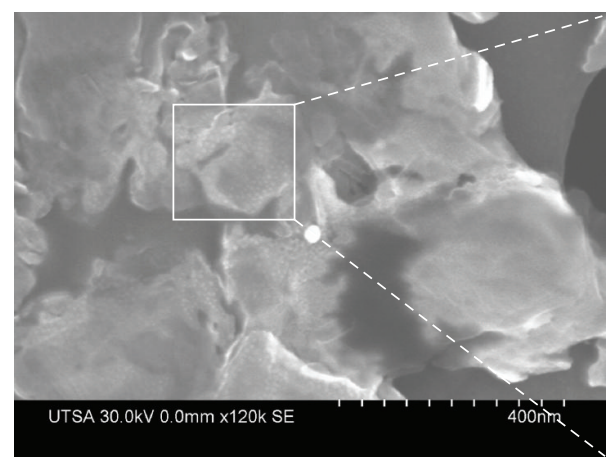

(a)

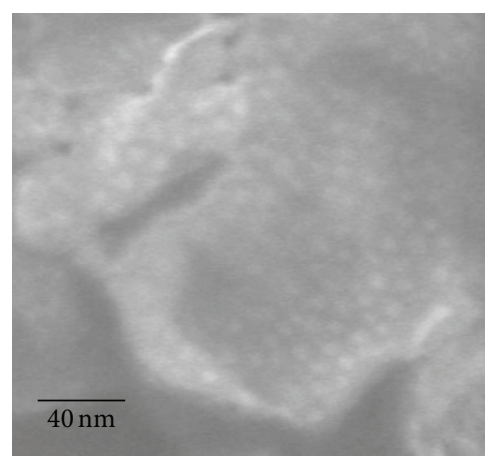

(b)

FIGURE 3: SEM micrograph of Au supported in zeolite (a) and zoom of a region (b).

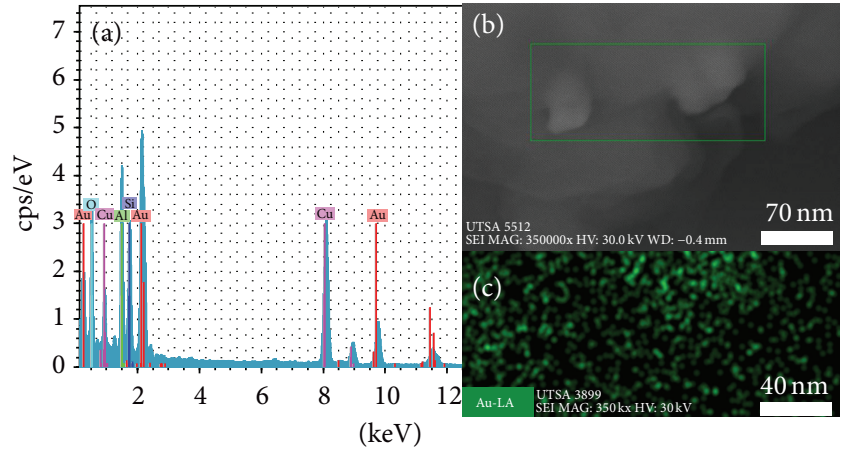

FIGURE 4: EDS analysis of Au supported on zeolite: (a) all zeolite components, (b) scan of a surface area, and (c) even distribution of $\mathrm{Au}$.

the $\mathrm{MB}$ solutions $(30 \mathrm{~mL})$, thus ensuring the contact between materials and solution. To initiate catalysis, the flasks were placed at a $10 \mathrm{~cm}$ distance from the LED light. Periodically, a volume of the $\mathrm{MB}$ solution was withdrawn in order to obtain its UV-Vis absorption spectrum. To quantify catalytic activity, the absorption decrease was monitored through time at $664 \mathrm{~nm}$. This wavelength corresponds to the strongest absorption for MB. This same procedure was followed in order to evaluate the catalytic activity mediated by sunlight. Solar irradiance for conducting experiments in Hermosillo, Sonora (Mexico), was $6 \mathrm{KWh} / \mathrm{m}^{2}$. As shown below, the results indicate that catalysis is more efficient regarding ncomp zeo-Au in the presence of sunlight. The characterization of these synthesized materials shows that nanomaterials are well below $2 \mathrm{~nm}$ and thus they may be located within its pores, which are a particular feature of these zeolites, thus enhancing catalytic efficiency.

The MB degradation percentage was calculated according to (1), where $A_{0}$ is absorbance at $t=0$ and $A$ is absorbance at time $t$. They were both measured at $664 \mathrm{~nm}$ :

$$
\% D=\left(\frac{A_{0}-A_{t}}{A_{0}}\right) \times 100
$$

To calculate $\mathrm{MB}$ photobleaching ratio, the LangmuirHinshelwood equation was used, which is expressed in (2) [31] as follows:

$$
\ln \frac{A}{A_{0}}=-k t
$$

where $k$ is the first-order rate constant of the photodegradation reaction, calculated as the slope of the regression plot $\ln \left(A / A_{0}\right)$ versus irradiation time $t\left(\ln \left(A / A_{0}\right)\right.$ versus $\left.t\right)$. All the absorbance values represented in the graph were obtained at $664 \mathrm{~nm}$.

3.6. Increased Rate of Catalysis (Sunlight). Photocatalysis under the sunlight is a technique that allows using a natural resource to increase the catalytic rate $[2,27,32]$. Sunlight is comprised of approximately $43 \%$ visible light, whereas only $5 \%$ of it is ultraviolet light and the rest belong to the range of infrared light [2]. The UV-Vis spectra shown in Figure 7 correspond to $\mathrm{MB}$ degradation as a function of time and under different light-exposure conditions in the presence of two different catalytic agents. In Figures 7(a) and 7(c) MB degradation is observed in contact with the zeolite matrix (without nanoparticles) when illuminated with white light LED and sunlight, respectively. Similarly, Figures 7(b) and 7(d) show MB degradation through heterogeneous catalysis with ncomp zeo-Au in the presence of white light (LED) and solar light, respectively.

Based on the maximum absorption of each spectrum at $664 \mathrm{~nm}$ in Figure 7, we obtained curves of MB degradation activity for each case. Figure 8 shows that degradation is faster under the sunlight (Figures 8(c) and 8(d)) in comparison with LED illumination (Figures 8(a) and 8(b)). Similarly, it shows that gold nanoparticles presence in zeolites accelerates MB degradation (Figures 8(b) and 8(d)) compared with the pure zeolite (Figures $8(\mathrm{a})$ and $8(\mathrm{c})$ ). Through comparison, the required time to achieve $50 \%$ of $\mathrm{MB}$ degradation is related for each curve. Figure 8(d) shows that the curve representing the more rapid $\mathrm{MB}$ degradation corresponds to the ncomp zeo-Au catalyst illuminated with sunlight, reaching a half degradation time of $t_{D 50}=11 \mathrm{~min}$. Conversely, the slowest 


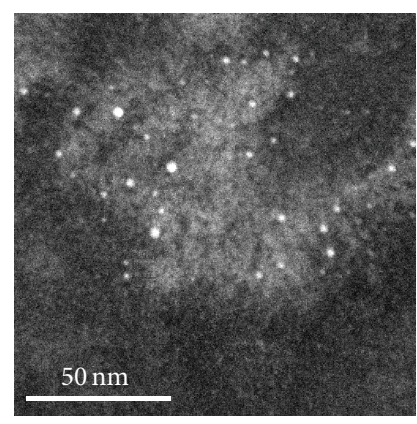

(a)

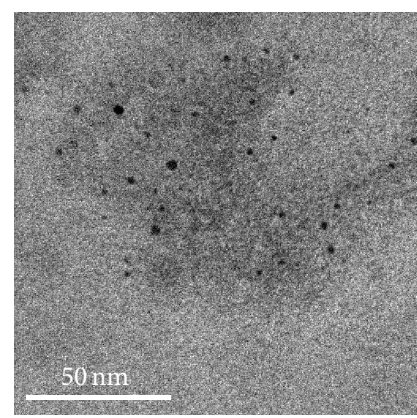

(e)

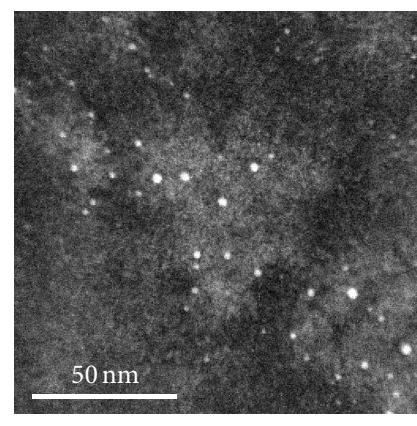

(b)

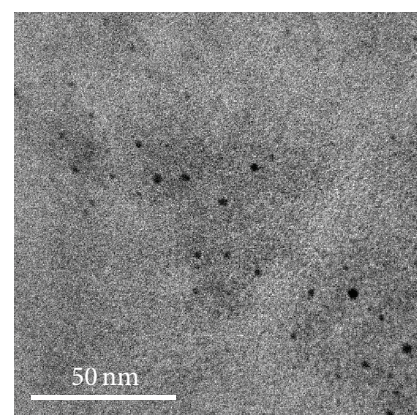

(f)

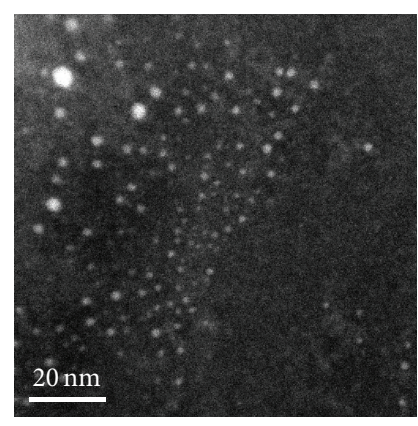

(c)

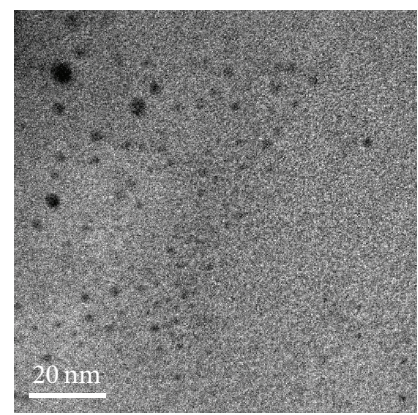

(g)

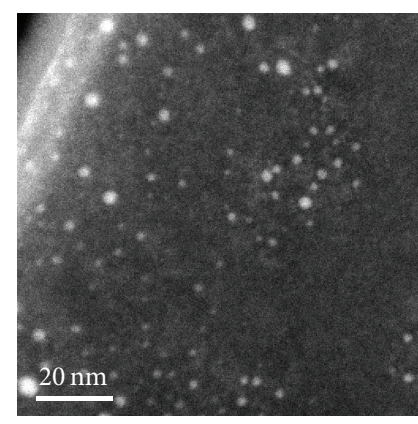

(d)

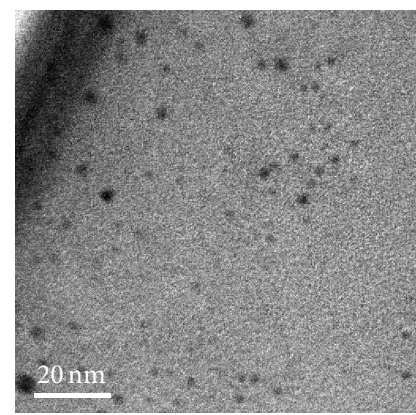

(h)

FIGURE 5: STEM micrographs of Au supported on zeolite: dark field (a-d) and bright field (e-h).

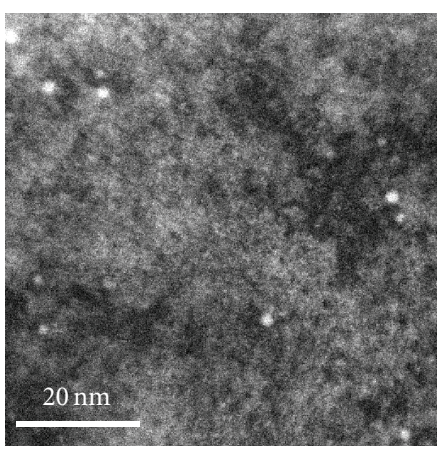

(a)

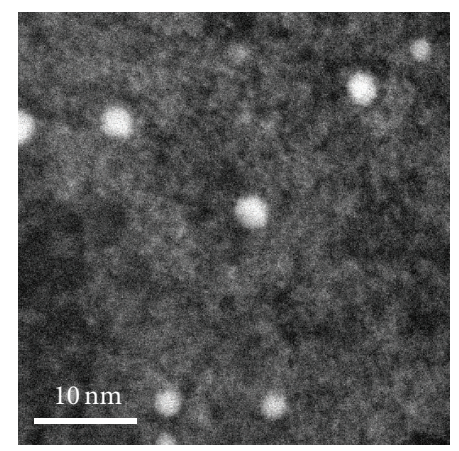

(b)

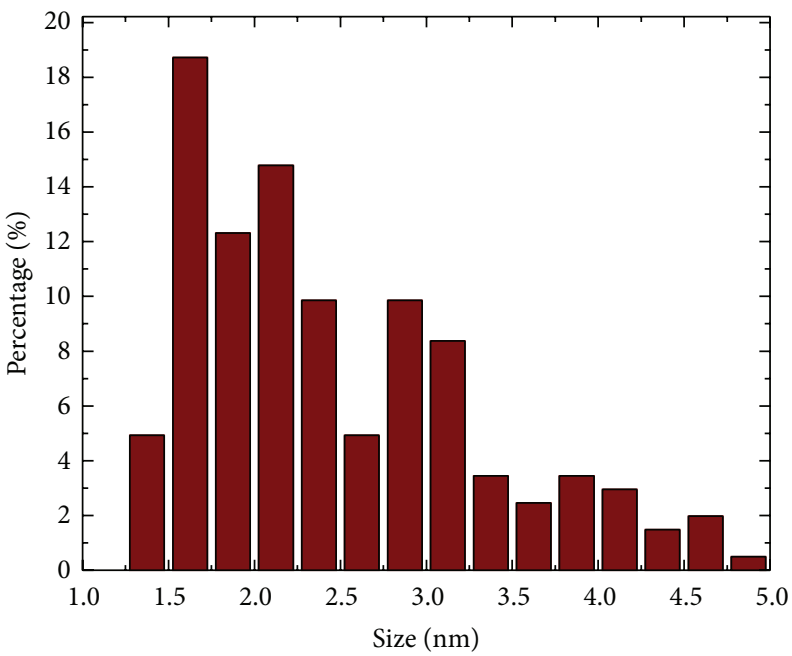

FIGURE 6: Higher magnification of STEM micrographs ( $\mathrm{a}$ and $\mathrm{b}$ ) and size distribution of Au nanoparticles supported in zeolite.

degradation rate corresponded to zeolite without nanoparticles (Figure $8(\mathrm{a})$ ), where $t_{D 50}=100 \mathrm{~min}$.

In Figure $9, \ln \left(A / A_{0}\right)$ versus $t$ plots were constructed, in which $k$ values as derived from (2) are observed. The graphs display the same relationship as in Figures 7 and 8. As shown in Figure 9, the data show a good linear correlation and the slopes calculated from the linear regression fit provided us with the value of the photodegradation reaction constants for each case. In Table 1 the most important data characterizing the reactions of $\mathrm{MB}$ photodegradation are summarized. It shows that the highest efficiency is obtained for $\mathrm{MB}$ degradation with ncomp zeo-Au matrix and photocatalyzed with sunlight. Gold nanoparticles present characteristic optical properties, particularly surface plasmon resonance (SPR) in the UV-Vis region. SPR effects arise from coupled oscillation of conductive electrons on the surface of nanoparticles caused by incident electromagnetic radiation. Recently, the presence of an absorption peak in the UV region associated with the interband excitation of electrons from level $5 d$ to $6 s p$ was demonstrated, and its intensity showed a close correlation with SPR, specially for nanoparticles with diameters below $2.5 \mathrm{~nm}$ [33]. Zhu et al. observed in $\mathrm{Au}$ nanoparticles supported on zeolites that the UV absorption peak produces a higher superficial photocurrent than the SPR 


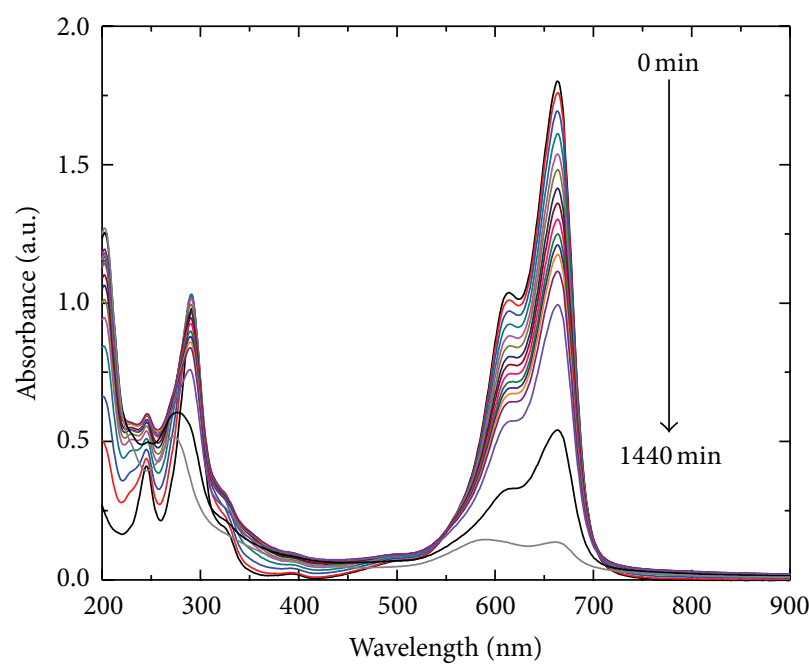

(a)

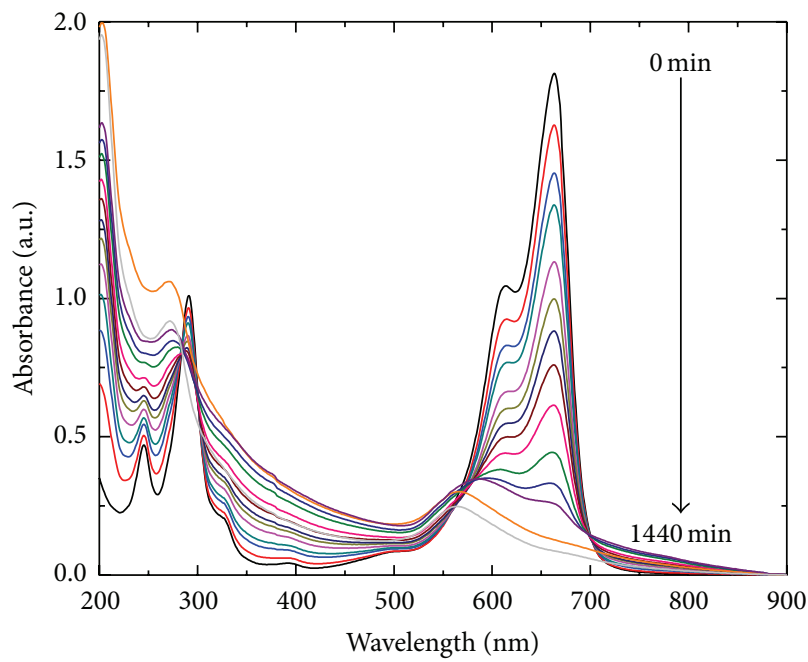

(c)

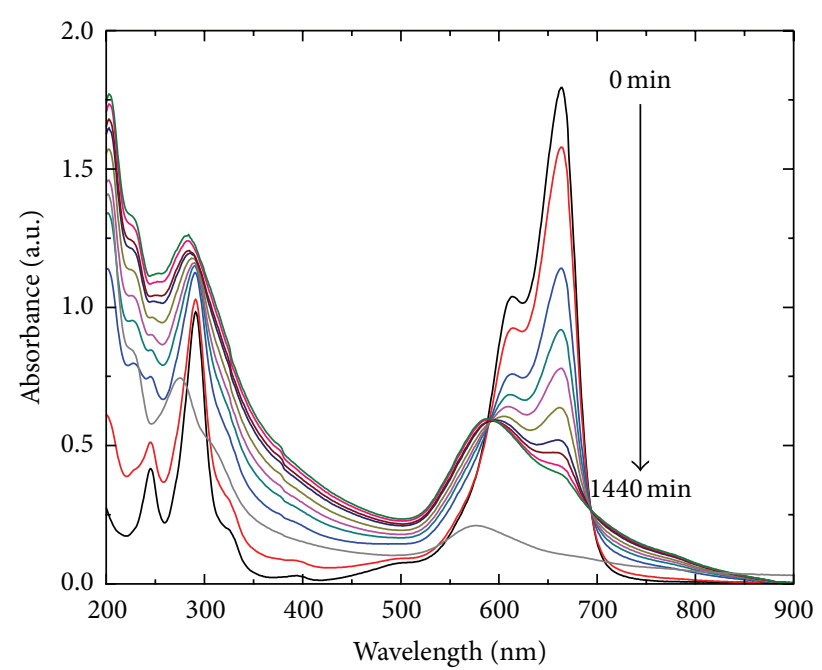

(b)

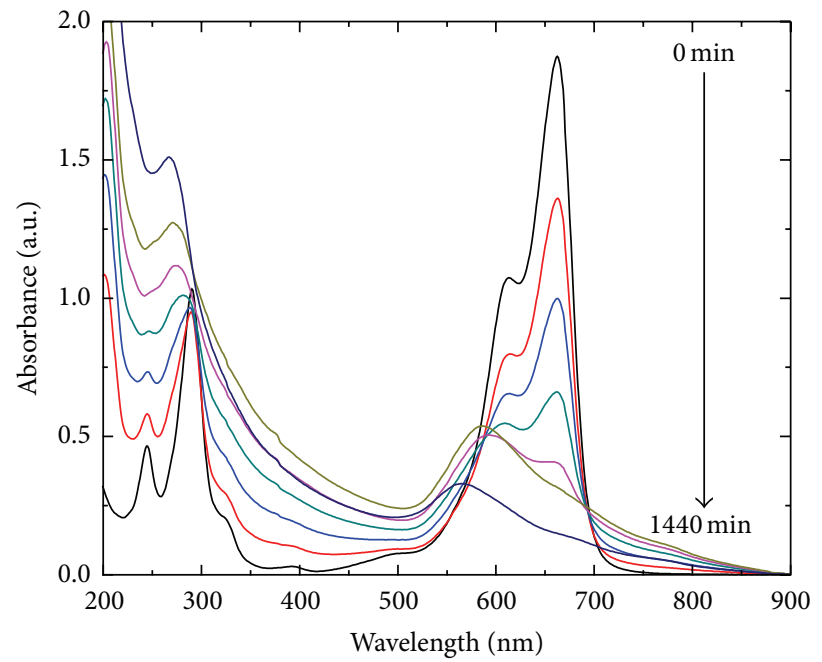

(d)

FIgURE 7: UV-Vis spectra of MB degradation. Natural zeolite: illuminated with LED (a) and sunlight (c); ncomp zeo-Au: illuminated with LED (b) and sunlight (d).

absorption in the Vis region [34]. The model proposed in this paper indicates that zeolites absorb oxygen in their pores and excited electrons on the surface of $\mathrm{Au}$ nanoparticles, causing formation of $\mathrm{O}_{2}{ }^{-}$species. As consequence of the electron transfer, a partially positive charge is generated in the $5 d$ level promoting electron transfer. Then, the Au nanoparticles take the equivalent electrons from absorbed organic molecules to neutralize their positive charge. The mechanism proposed supports our observations of a higher degradation rate of methylene blue with sunlight in comparison with white light. As control, the same catalytic reaction was performed protected from light. After $24 \mathrm{~h}$, the control showed less than $10 \%$ degradation of methylene blue, confirming that the catalytic reaction is dependent on light.

Furthermore, the $z$ potential was also measured for both pure zeolite and ncomp zeo-Au systems and the respective values are shown in Table 2 . In both cases, the system had a substantial negative electrostatic charge interacting with the positive charge of $\mathrm{MB}$ and causing electrostatic interactions between dye and the catalyst molecules. This may be a determinative factor for the efficient catalysis mechanism. It should be noted that the results obtained for the catalytic activity do not include the addition of compounds enhancing the degradation reaction, such as sodium borohydride. However, it was assumed that residual ascorbic acid molecules derived from the in situ synthesis of gold nanoparticles remain in the ncomp zeo-Au. Thus, the addition of another reducing agent is not necessary when catalyzing MB degradation.

\section{Conclusions}

The processes and methods implemented in these systems intended to clean water include green materials only: ascorbic acid and natural zeolites. The production of a nanomaterial as small as possible, encompassing a larger surface area, is 


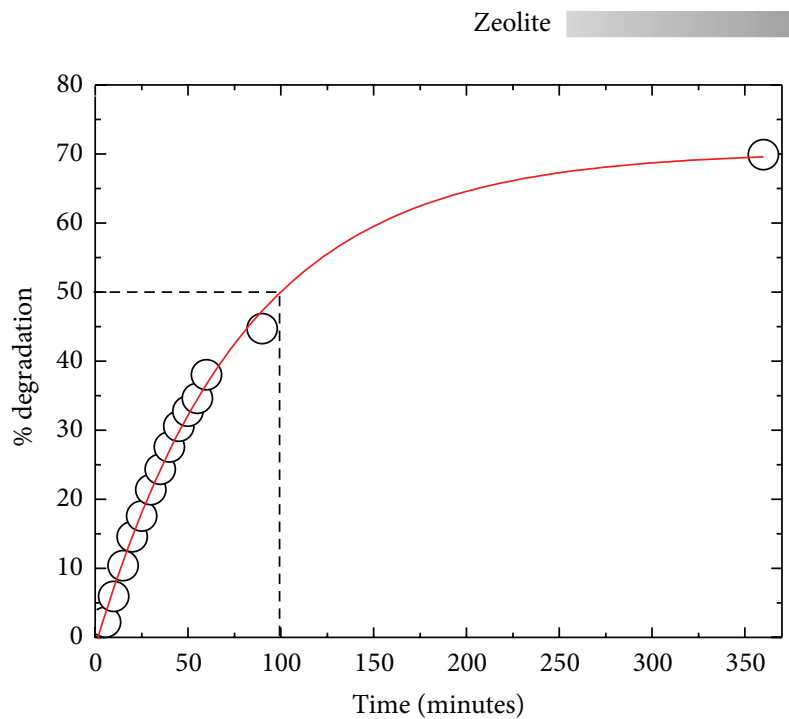

(a)

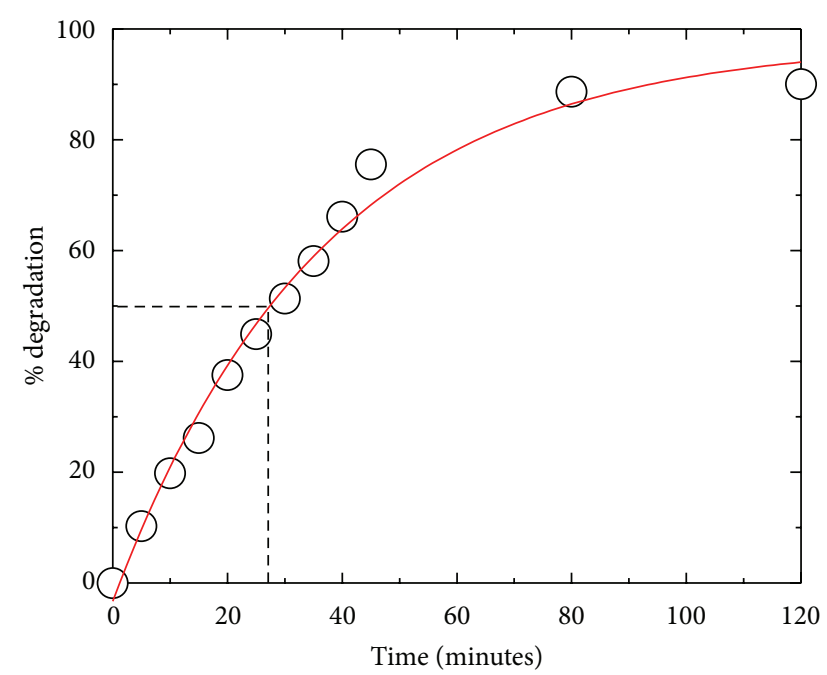

(c)

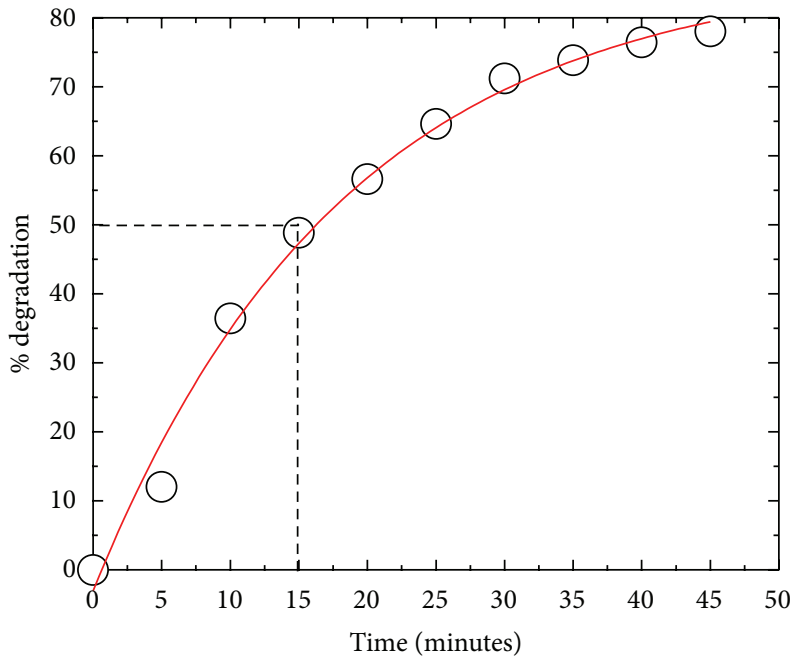

(b)

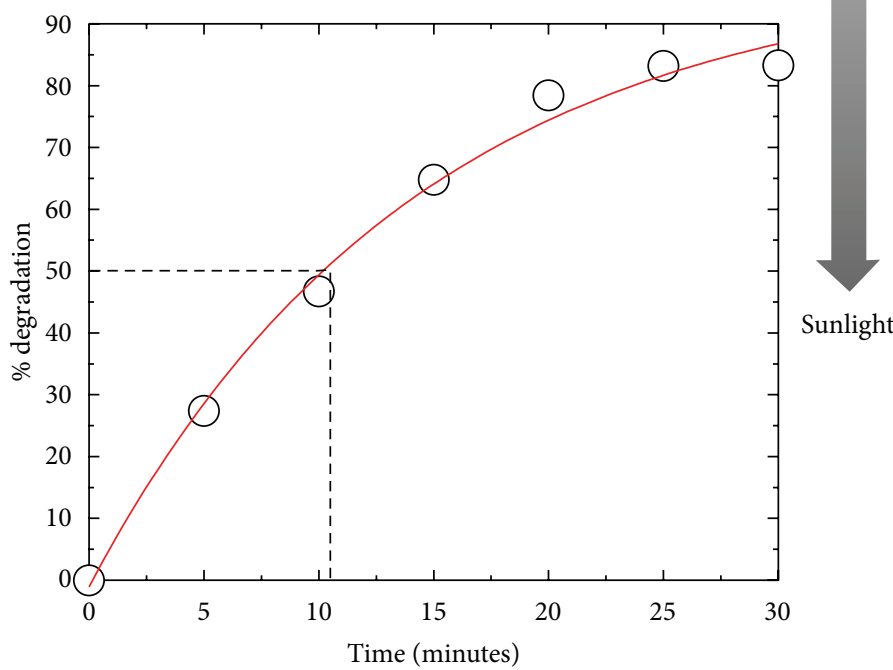

(d)

FIgURE 8: Percentage of MB degradation and the time period to achieve 50\% degradation. Natural zeolite: illuminated with LED (a) and sunlight (c); ncomp zeo-Au: illuminated with LED (b) and sunlight (d).

TABLE 1: Parameters for each system: percentage of total degradation $\left(\% D_{t}\right)$, degradation ratio $(k)$, and time to achieve $50 \%$ degradation $\left(t_{D 50}\right)$.

\begin{tabular}{lccc}
\hline Sample (0.15 g) & $\% D_{t \rightarrow \infty}$ & $k\left(\mathrm{~min}^{-1}\right)$ & $t_{D 50}(\mathrm{~min})$ \\
\hline Zeo (LED light) & 70 & 0.008 & 100 \\
Zeo-Au (LED light) & 87 & 0.04 & 15 \\
Zeo (sunlight) & 100 & 0.02 & 26 \\
Zeo-Au (sunlight) & 100 & 0.07 & 11 \\
\hline
\end{tabular}

needed in order to obtain an enhanced catalytic activity. In this case, the characteristic matrix pores of the natural zeolite can be employed as cavities in which nanomaterials (particles or clusters) are synthesized and confined. The results obtained by transmission electron microscopy in scanning
TABLE 2: Physicochemical properties: $\mathrm{pH}$, conductivity, and zeta potential of natural zeolite and ncomp zeo-Au.

\begin{tabular}{lccc}
\hline Sample $(0.15 \mathrm{~g})$ & $\mathrm{pH}$ & $\begin{array}{c}\text { Conductivity } \\
(\mu \mathrm{S} / \mathrm{cm}) \\
T=25^{\circ} \mathrm{C}\end{array}$ & $z$ potential $(\mathrm{mV})$ \\
\hline Zeo & 6.914 & 28 & -37 \\
Zeo-Au & 5.973 & 103.7 & -29 \\
\hline
\end{tabular}

mode (STEM) using the synthesized samples sectioned in nanomaterials by ultramicrotome confirm that nanoparticles under $2 \mathrm{~nm}$ are housed inside the pores of the zeolites.

This synthesis is considered as sustainable chemistry as it is performed at room temperature $\left(20^{\circ} \mathrm{C}-25^{\circ} \mathrm{C}\right)$ and using ascorbic acid as reducing agent; therefore it does not generate 


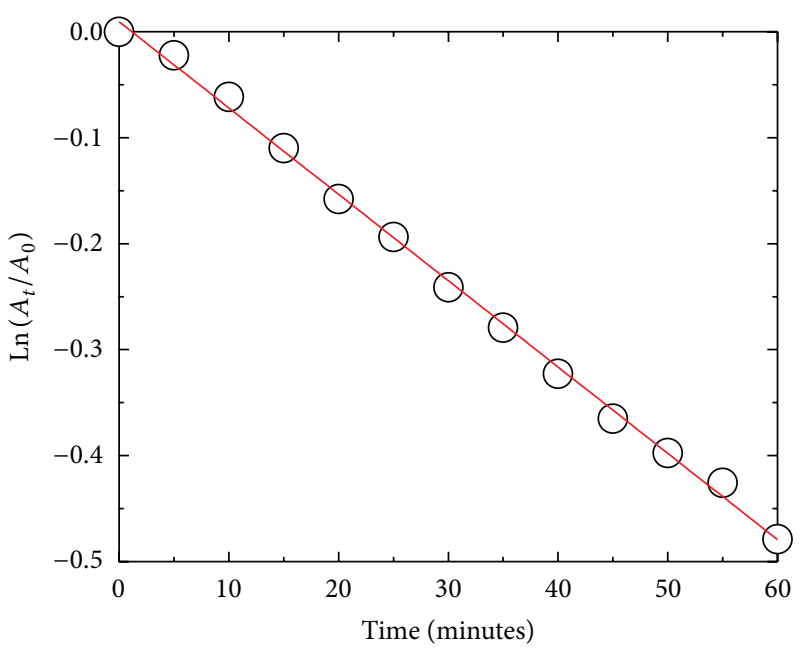

(a)

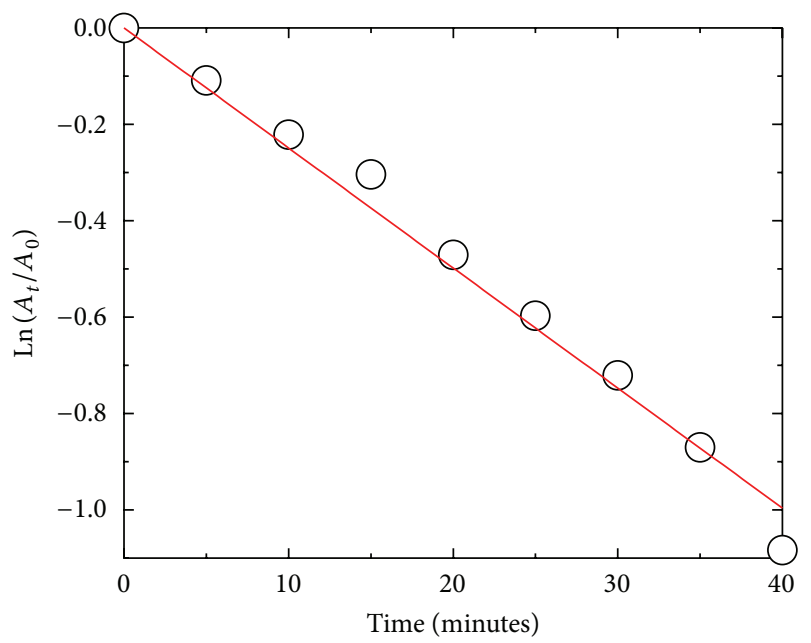

(c)

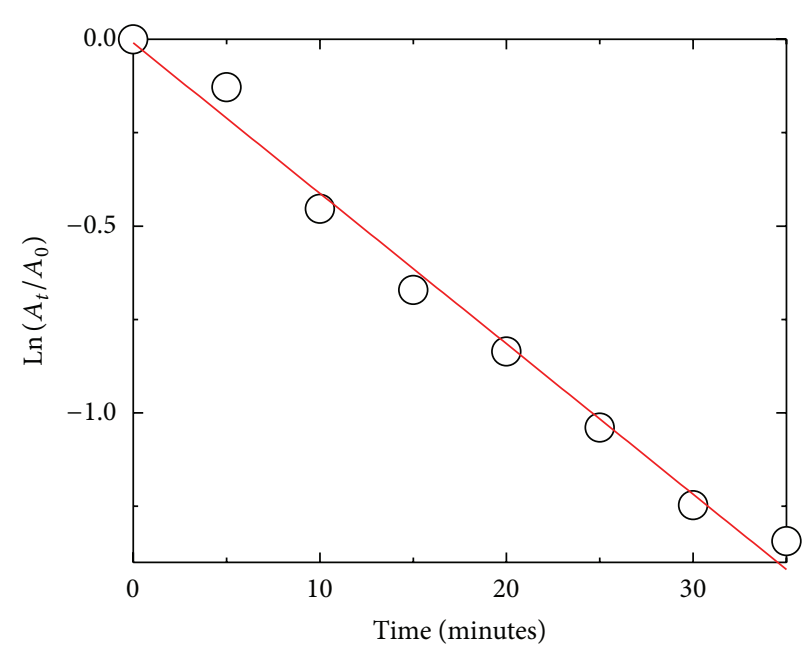

(b)

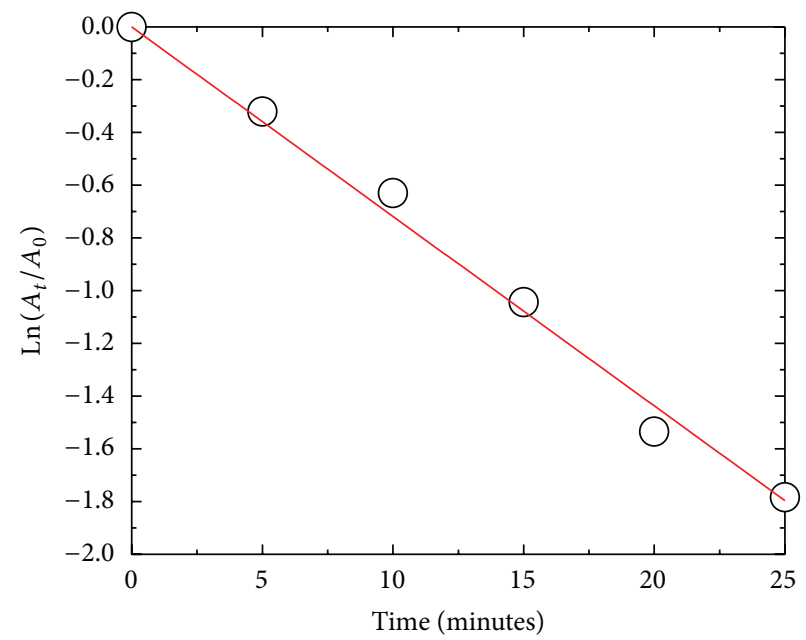

(d)

FIGURE 9: Ratio of MB degradation in samples; natural zeolite: illuminated with LED (a) and sunlight (c); ncomp zeo-Au: illuminated with LED (b) and sunlight (d).

environmental pollution. The degradation of $\mathrm{MB}$ by the synthesized nanomaterials is performed at room temperature without using a reducing agent, for example, sodium borohydride $\left(\mathrm{NaBH}_{4}\right)$. Therefore, this method reduces the cost of the process and the environmental impact. The catalysis presented in this study is of the heterogeneous type; thus ncomp zeo-Au matrix can be reused in other catalytic cycles by simply removing the container in which the material is confined.

\section{Competing Interests}

The authors declare that there is no conflict of interests regarding the publication of this paper.

\section{Acknowledgments}

All authors thank The University of Texas at San Antonio (UTSA) for access to facilities and the use of STEM and X-ray diffraction and Dr. Fernando Hinojosa and Ms. Kareen Krizzan Encinas Soto of Departamento de Ingeniería Química y Metalurgia, Universidad de Sonora, for access and the use of Gemini VII 2390 (Micrometrics). They also thank CONACYT for the Project PROINNOVA no. 221252 and PRODEP for the Project DSA/103.5/14/10945.

\section{References}

[1] N. Linares, A. M. Silvestre-Albero, E. Serrano, J. SilvestreAlbero, and J. García-Martínez, "Mesoporous materials for clean energy technologies," Chemical Society Reviews, vol. 43, no. 22, pp. 7681-7717, 2014.

[2] S. Sarina, E. R. Waclawik, and H. Zhu, "Photocatalysis on supported gold and silver nanoparticles under ultraviolet and visible light irradiation," Green Chemistry, vol. 15, no. 7, pp. 18141833, 2013.

[3] J. Zhang, F.-T. Hu, Q.-Q. Liu, X. Zhao, and S.-Q. Liu, “Application of heterogenous catalyst of tris $(1,10)$-phenanthroline 
iron(II) loaded on zeolite for the photo-Fenton degradation of methylene blue," Reaction Kinetics, Mechanisms and Catalysis, vol. 103, no. 2, pp. 299-310, 2011.

[4] R. Han, J. Zhang, P. Han, Y. Wang, Z. Zhao, and M. Tang, "Study of equilibrium, kinetic and thermodynamic parameters about methylene blue adsorption onto natural zeolite," Chemical Engineering Journal, vol. 145, no. 3, pp. 496-504, 2009.

[5] J. Shi, "On the synergetic catalytic effect in heterogeneous nanocomposite catalysts," Chemical Reviews, vol. 113, no. 3, pp. 2139-2181, 2013.

[6] M. A. El-Sayed, "Some interesting properties of metals confined in time and nanometer space of different shapes," Accounts of Chemical Research, vol. 34, no. 4, pp. 257-264, 2001.

[7] V. Ramamurthy, "Controlling photochemical reactions via confinement: zeolites," Journal of Photochemistry and Photobiology C: Photochemistry Reviews, vol. 1, no. 2, pp. 145-166, 2000.

[8] T. Sen, S. Jana, S. Koner, and A. Patra, "Efficient energy transfer between confined dye and Y-zeolite functionalized Au nanoparticles," The Journal of Physical Chemistry C, vol. 114, no. 46, pp. 19667-19672, 2010.

[9] K. Ravikumar, B. Deebika, and K. Balu, "Decolourization of aqueous dye solutions by a novel adsorbent: application of statistical designs and surface plots for the optimization and regression analysis," Journal of Hazardous Materials, vol. 122, no. 1-2, pp. 75-83, 2005.

[10] S. T. H. Sherazi, R. A. Soomro, S. Uddin, and N. Memon, "Synthesis and characterizations of highly efficient copper nano particles and their use in ultra fast catalytic degradation of organic dyes," Advanced Materials Research, vol. 829, pp. 93-99, 2014.

[11] M. J. Ndolomingo and R. Meijboom, "Kinetic analysis of catalytic oxidation of methylene blue over $\gamma-\mathrm{Al}_{2} \mathrm{O}_{3}$ supported copper nanoparticles," Applied Catalysis A: General, vol. 506, pp. 33-43, 2015.

[12] P. Ncube, N. Bingwa, H. Baloyi, and R. Meijboom, "Catalytic activity of palladium and gold dendrimer-encapsulated nanoparticles for methylene blue reduction: a kinetic analysis," Applied Catalysis A: General, vol. 495, pp. 63-71, 2015.

[13] M. Rafatullah, O. Sulaiman, R. Hashim, and A. Ahmad, "Adsorption of methylene blue on low-cost adsorbents: a review," Journal of Hazardous Materials, vol. 177, no. 1-3, pp. 7080, 2010.

[14] Y. Li, Q. Du, T. Liu et al., "Comparative study of methylene blue dye adsorption onto activated carbon, graphene oxide, and carbon nanotubes," Chemical Engineering Research and Design, vol. 91, no. 2, pp. 361-368, 2013.

[15] J. Fu, Z. Chen, M. Wang et al., "Adsorption of methylene blue by a high-efficiency adsorbent (polydopamine microspheres): kinetics, isotherm, thermodynamics and mechanism analysis," Chemical Engineering Journal, vol. 259, pp. 53-61, 2015.

[16] S. Sohrabnezhad and A. Pourahmad, "Comparison absorption of new methylene blue dye in zeolite and nanocrystal zeolite," Desalination, vol. 256, no. 1-3, pp. 84-89, 2010.

[17] O. Korkuna, R. Leboda, J. Skubiszewska-Zięba, T. Vrublevs'ka, V. M. Gun'ko, and J. Ryczkowski, "Structural and physicochemical properties of natural zeolites: clinoptilolite and mordenite," Microporous and Mesoporous Materials, vol. 87, no. 3, pp. 243254, 2006.

[18] S. Hashimoto, "Zeolite photochemistry: impact of zeolites on photochemistry and feedback from photochemistry to zeolite science," Journal of Photochemistry and Photobiology C: Photochemistry Reviews, vol. 4, no. 1, pp. 19-49, 2003.
[19] R. Xu, W. Pang, J. Yu, Q. Huo, and J. Chen, Chemistry of Zeolites and Related Porous Materials: Synthesis and Structure, John Wiley \& Sons, 2009.

[20] K. B. Narayanan and H. H. Park, "Homogeneous catalytic activity of gold nanoparticles synthesized using turnip (Brassica rapa L.) leaf extract in the reductive degradation of cationic azo dye," Korean Journal of Chemical Engineering, vol. 32, no. 7, pp. 1273-1277, 2015.

[21] V. S. Suvith and D. Philip, "Catalytic degradation of methylene blue using biosynthesized gold and silver nanoparticles," Spectrochimica Acta A: Molecular and Biomolecular Spectroscopy, vol. 118, pp. 526-532, 2014.

[22] V. A. Kumar, T. Uchida, T. Mizuki et al., "Synthesis of nanoparticles composed of silver and silver chloride for a plasmonic photocatalyst using an extract from a weed Solidago altissima (goldenrod)," Advances in Natural Sciences: Nanoscience and Nanotechnology, vol. 7, no. 1, Article ID 015002, 2016.

[23] X. Zhang, X. Ke, A. Du, and H. Zhu, "Plasmonic nanostructures to enhance catalytic performance of zeolites under visible light," Scientific Reports, vol. 4, article 3805, pp. 1-6, 2014.

[24] M. Arvand, S. Sohrabnezhad, M. F. Mousavi, M. Shamsipur, and M. A. Zanjanchi, "Electrochemical study of methylene blue incorporated into mordenite type zeolite and its application for amperometric determination of ascorbic acid in real samples," Analytica Chimica Acta, vol. 491, no. 2, pp. 193-201, 2003.

[25] E. Sanatgar-Delshade, A. Habibi-Yangjeh, and M. KhodadadiMoghaddam, "Hydrothermal low-temperature preparation and characterization of $\mathrm{ZnO}$ nanoparticles supported on natural zeolite as a highly efficient photocatalyst," Monatshefte für Chemie-Chemical Monthly, vol. 142, no. 2, pp. 119-129, 2011.

[26] X. Chen, Z. Zheng, X. Ke et al., "Supported silver nanoparticles as photocatalysts under ultraviolet and visible light irradiation," Green Chemistry, vol. 12, no. 3, pp. 414-419, 2010.

[27] S. Sarina, H. Zhu, E. Jaatinen et al., "Enhancing catalytic performance of palladium in gold and palladium alloy nanoparticles for organic synthesis reactions through visible light irradiation at ambient temperatures," Journal of the American Chemical Society, vol. 135, no. 15, pp. 5793-5801, 2013.

[28] C. Lin, K. Tao, D. Hua, Z. Ma, and S. Zhou, "Size effect of gold nanoparticles in catalytic reduction of p-nitrophenol with $\mathrm{NaBH}_{4}$," Molecules, vol. 18, no. 10, pp. 12609-12620, 2013.

[29] Sirajuddin, A. Nafady, H. I. Afridi, S. Sara, A. Shah, and A. Niaz, "Direct synthesis and stabilization of Bi-sized cysteinederived gold nanoparticles: reduction catalyst for methylene blue," Journal of the Iranian Chemical Society, vol. 8, no. 1, pp. S34-S43, 2011.

[30] J.-H. Kim, K. M. Twaddle, J. Hu, and H. Byun, "Sunlightinduced synthesis of various gold nanoparticles and their heterogeneous catalytic properties on a paper-based substrate," ACS Applied Materials and Interfaces, vol. 6, no. 14, pp. 11514$11522,2014$.

[31] A. Nezamzadeh-Ejhieh and H. Zabihi-Mobarakeh, "Heterogeneous photodecolorization of mixture of methylene blue and bromophenol blue using CuO-nano-clinoptilolite," Journal of Industrial and Engineering Chemistry, vol. 20, no. 4, pp. 14211431, 2014.

[32] A. Nezamzadeh-Ejhieh and S. Hushmandrad, "Solar photodecolorization of methylene blue by $\mathrm{CuO} / \mathrm{X}$ zeolite as a heterogeneous catalyst," Applied Catalysis A: General, vol. 388, no. 1-2, pp. 149-159, 2010. 
[33] B. Balamurugan and T. Maruyama, "Evidence of an enhanced interband absorption in Au nanoparticles: size-dependent electronic structure and optical properties," Applied Physics Letters, vol. 87, no. 14, Article ID 143105, 2005.

[34] H. Zhu, X. Chen, Z. Zheng et al., "Mechanism of supported gold nanoparticles as photocatalysts under ultraviolet and visible light irradiation," Chemical Communications, vol. 48, pp. 75247526, 2009. 

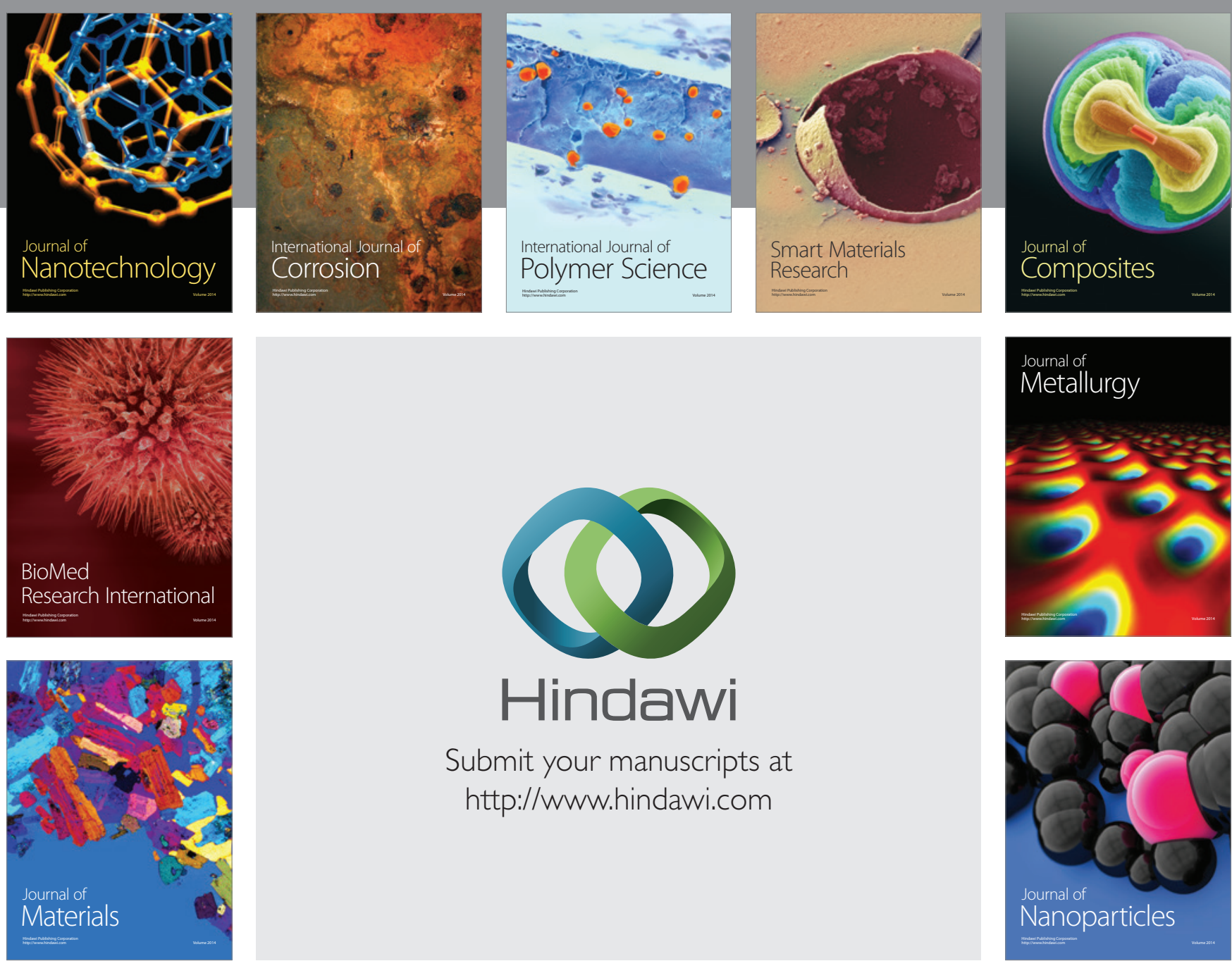

\section{Hindawi}

Submit your manuscripts at

http://www.hindawi.com

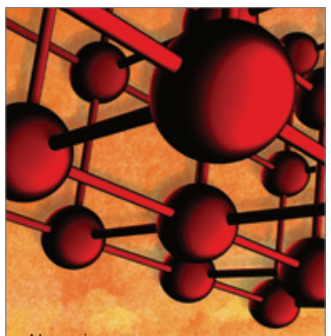

Materials Science and Engineering
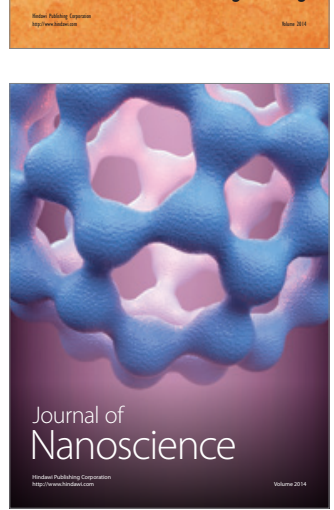
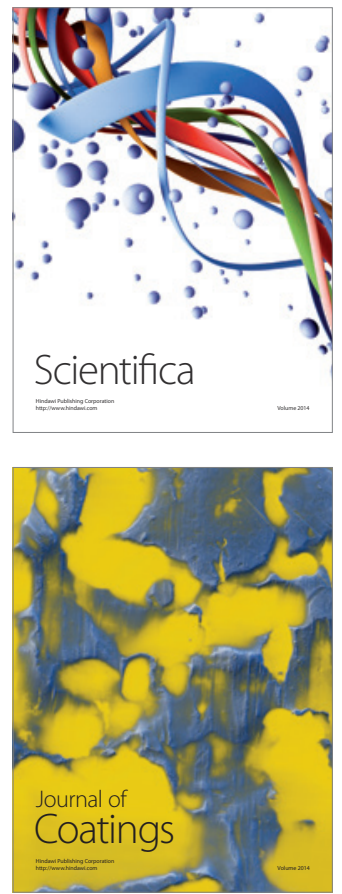
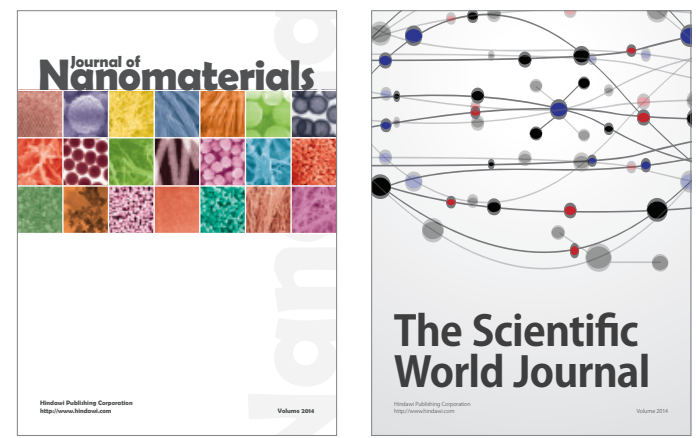

The Scientific World Journal
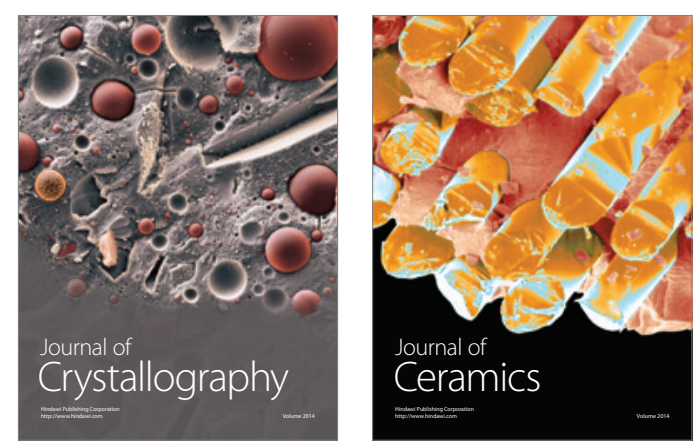
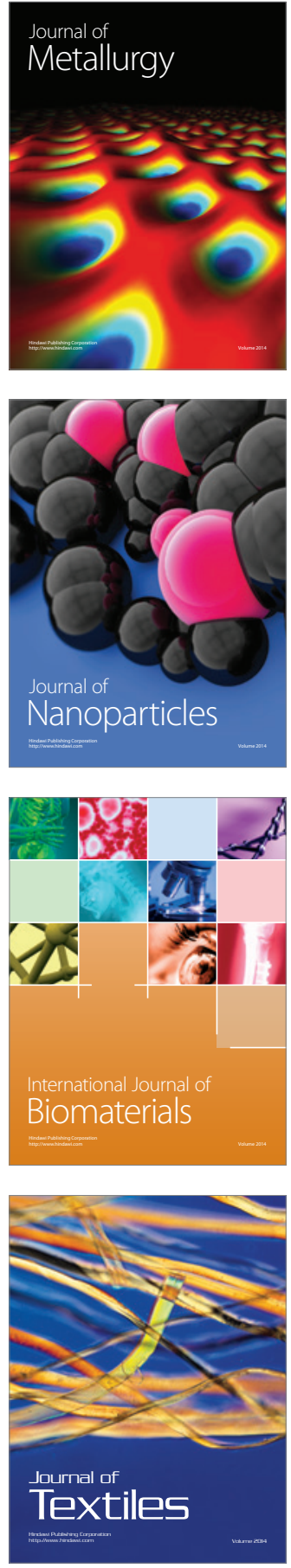